Canadian Oncology

Nursing Journal

Revue canadienne

de soins infirmiers

en oncologie

Volume 31, Issue 3 • Summer 2021

elSSN: 2368-8076 


\title{
Concept d'adaptation chez les conjoints de femmes iraniennes atteintes du cancer du sein : étude qualitative basée sur une approche phénoménologique
}

\author{
par Marzieh Jahani Sayad Noveiri, Farshid Shamsaei, Masoud Khodaveisi, Zohreh Vanaki, Lily Tapak
}

RÉSUMÉ

Introduction : Le cancer du sein provoque une crise multidimensionnelle qui ébranle non seulement la patiente, mais aussi le conjoint et les autres membres de sa famille. Il convient donc d'étudier l'adaptation à ce phénomène, qui constitue l'une des principales difficultés pour les proches. En comprenant mieux comment les conjoints de femmes atteintes d'un cancer du sein font face à cette crise, ces derniers pourraient mieux prendre soin de leurs épouses et améliorer leur qualité de vie.

\section{AUTEURS}

Marzieh Jahani Sayad Noveiri, Comité de recherche étudiante, Université des sciences médicales de Hamadan, Hamadan, Iran; jahanimzh@gmail.com

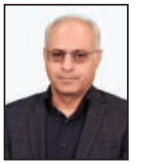

Farshid Shamsaei, Centre de recherche en soins de la mère et de l'enfant, Université des sciences médicales de Hamadan, Hamadan, Iran; (auteur-ressource) shamsaei68@yahoo.com

Masoud Khodaveisi, Centre de recherche sur les maladies chroniques (soins à domicile), Département des soins infirmiers communautaires, Université des sciences médicales de Hamadan, Hamadan, Iran; khodaveisi2000@yahoo.com

Zohreh Vanaki, Département des sciences infirmières, Faculté des sciences médicales, Université Tarbiat Modares, Téhéran, Iran; vanaki_z@modares.ac.ir

Lily Tapak, Département de biostatistique, École de santé publique, Centre de recherche sur la modélisation des maladies non transmissibles, Université des sciences médicales de Hamadan, Hamadan, Iran; tapak@umsha.ac.ir

Auteur-ressource : Farshid Shamsaei, École de sciences infirmières et pratique sage-femme, Université des sciences médicales de Hamadan, Shahid Fahmideh Bulv., Hamadan 65178, Iran

Tél. : +98-81-38381941; Téléc. : +98-81-38381942

Courriel : shamsaei68@yahoo.com

Remerciements : Cet article résulte d'une thèse de doctorat soutenue par l'Université des sciences médicales de Hamadan. Les auteurs souhaitent exprimer leur gratitude pour le soutien financier accordé par le vice-recteur du département de recherche et de technologie de l'Université des sciences médicales de Hamadan.

Déclaration de conflit d'intérêts : Aucun conflit d'intérêts potentiel n'a été déclaré par les auteurs.

Financement: Ces travaux ont été financés par l'Université des sciences médicales de Hamadan, Hamadan, Iran ( $n^{\circ}$ 9710256388)
But de l'étude : L'étude visait à explorer le concept d'adaptation à partir d'expériences vécues par les conjoints de femmes souffrant d'un cancer du sein.

Méthodologie : Cette étude qualitative a été réalisée en 2019 selon une approche phénoménologique dans les villes de Hamadan et Rasht, en Iran. Le groupe de participants comptait 20 conjoints de femmes atteintes du cancer du sein, recrutés grâce à une méthode d'échantillonnage par choix raisonné. Les données ont été recueillies au moyen d'entretiens non structurés en personne; elles ont été analysées à l'aide de la méthode phénoménologique en six étapes de van Manen.

Résultats : Les expériences vécues par les participants ont montré que le phénomène d'adaptation des conjoints au cancer du sein de leur femme se décline en cinq thèmes : souffrance émotionnelle, fardeau des soins, maintien de la cohésion familial, incertitude de l'avenir et sentiment de perte du concept de soi. La compréhension $d u$ concept d'adaptation chez les conjoints de femmes atteintes $d u$ cancer du sein dans les stratégies de santé peut aider les femmes à bien s'adapter, tout en aidant les professionnels à adopter des mesures efficaces dans le domaine des soins médicaux, pour les patientes et leurs conjoints.

Mots-clés : cancer du sein, adaptation, conjoints, phénoménologie

\section{INTRODUCTION}

T e cancer du sein est l'un des cancers les plus répandus _dans la plupart des pays, y compris en Iran, où la prévalence de la maladie est estimée à 24,6 pour 100000 personnes (Abachizadeh et al., 2018). Chaque année, ce cancer bouleverse la vie de milliers de conjoints iraniens. Vivre avec cette maladie est considéré comme une expérience tragique et critique entraînant des réactions psychologiques comme la non-acceptation, le déni, la colère, le stress et l'anxiété, l'incertitude et le désespoir chez les femmes et les familles, plus particulièrement pour les conjoints (Braun et al., 2007).

Le cancer du sein est le type de cancer le plus courant chez la femme; il se classe en effet au deuxième rang de tous les cancers, de sorte que plus de 2,1 millions de nouveaux cas de cancer du sein ont été enregistrés par le Centre mondial des statistiques sur le cancer en 2018 (Bray et al., 2018; Momenimovahed et Salehiniya, 2019). Après le cancer du poumon, le cancer du sein est la seconde cause de décès 
liés au cancer (15,4\%) dans les pays développés. Il arrive au cinquième rang en Iran, avec un taux de mortalité estimé à 14,2 \% (Nafissi et al., 2018). Malgré les avancées en matière de diagnostic et d'équipement de traitement du cancer du sein en Iran, les rapports indiquent que la prévalence de la maladie augmente toujours (Montazeri et al., 2008; Nasiri, Taleghani et Irajpour, 2016).

Le cancer du sein peut avoir des conséquences directes sur les conjoints des patientes (Braun et al., 2007; Yusoff et al., 2012). La détresse des conjoints de femmes ayant un cancer du sein fait partie des effets amplement observés. Certaines études ont même fait état d'une détresse plus importante chez les conjoints que chez les patientes (Braun et al., 2007; Northouse et al., 2000). Cela s'expliquerait par le fait qu'ils assument plus de responsabilités en tant que soignants durant le traitement, et aussi que leurs propres besoins sont négligés par les proches et le personnel médical (Northouse et al., 2000; Soothill et al., 2003).

Léquipe de recherche s'est penchée sur les enjeux relatifs au soutien social, aux relations conjugales et aux stratégies d'adaptation des conjoints de patientes atteintes d'un cancer du sein. Les études indiquent généralement que le cancer du sein transforme le cadre de vie des conjoints, dont les problèmes tournent principalement autour de questions comme la satisfaction conjugale, la communication et le soutien interpersonnel, qui renvoient aux efforts qu'ils doivent déployer pour préserver l'environnement familial (Dorval et al., 2005; Yusoff et al., 2012). Les conjoints doivent s'adapter aux circonstances pour être en mesure de soutenir leur partenaire malade, d'éloigner le stress du nid familial et de s'occuper des tâches du quotidien.

L'adaptation représente l'une des plus grandes difficultés rencontrées par les conjoints, car non seulement le cancer perturbe la vie de famille, mais il affecte aussi leur statut socioéconomique, en plus de créer divers problèmes dans la relation de couple et les rôles qui leur incombent (Gao et al., 2019; Lopes et al., 2018; Younes Barani, et al., 2019).

L'adaptation requiert l'utilisation de stratégies cognitivo-comportementales de gestion du stress qui misent, entre autres, sur les émotions et la résolution de problèmes. Les stratégies axées sur la résolution de problèmes visent à contrôler les sources de stress, alors que celles basées sur les émotions visent à canaliser les pensées et les émotions (Fink, 2016; Folkman et Lazarus, 1988; Mehrabi et al., 2016). Le processus d'adaptation se déroule différemment pour chacun, et à un rythme qui lui est propre (Cebeci et al., 2012; Ghaffari et al., 2014). Les hommes dont la conjointe combat un cancer du sein ont recours à différentes stratégies d'adaptation au cours des phases d'autocontrôle et d'autogestion. L'acceptation, même si chacun la vit à sa manière, est l'une des stratégies d'adaptation habituellement vécue (Kershaw et al., 2004; Yeung et al., 2018). Le vécu des personnes atteintes du cancer du sein et de leurs conjoints diffère du vécu des gens qui n'ont pas traversé cette épreuve, tout comme les expériences d'adaptation des conjoints diffèrent de celles des patientes. Comprendre le phénomène d'adaptation contribuera à faire ressortir les facteurs influençant l'adaptation des conjoints, ce qui facilitera aussi le traitement des patientes et aidera le personnel médical à intervenir efficacement.

Une revue de la littérature portant sur l'adaptation des conjoints de femmes atteintes du cancer du sein a relevé certaines études qui se sont intéressées aux besoins de ces hommes (Sajadian et al., 2015). Cependant, aucune étude qualitative sur les expériences d'adaptation des conjoints iraniens n'a pu être trouvée. Compte tenu des différences observées dans la société iranienne quant à l'approche culturelle, aux croyances et aux valeurs véhiculées, l'approche qualitative apparaît comme la meilleure méthode pour examiner les expériences d'adaptation. La présente étude qualitative a donc été menée afin d'obtenir une compréhension approfondie des expériences d'adaptation des conjoints de femmes atteintes d'un cancer du sein en Iran.

\section{MÉTHODOLOGIE}

\section{Devis de l'étude}

Cette étude qualitative utilise une approche interprétative phénoménologique de type herméneutique. Il s'agit d'une méthodologie qualitative utilisée pour examiner et comprendre l'expérience subjective d'individus et qui privilégie une approche idiographique selon laquelle des cas individuels détaillés sont étudiés pour élaborer des observations plus générales (Paley, 2018; Walker, 2007; Xiong et al., 2016). Cette méthodologie permet d'approfondir la compréhension de l'expérience humaine par l'entremise de la description et de l'interprétation. Grâce à une approche herméneutique de l'analyse, les principes de la philosophie phénoménologique de Heidegger ont permis d'explorer la réalité des conjoints qui doivent composer avec le cancer du sein de leur femme, en Iran.

\section{Participants et contexte}

Le groupe de participants était formé de 20 hommes dont la conjointe était atteinte du cancer du sein. Ils ont été sélectionnés grâce à une méthode d'échantillonnage par choix raisonné, parmi les patientes des hôpitaux de Hamadan et de Rasht, en Iran. Pour être choisi, chaque participant devait être conjoint et soignant d'une femme dont le diagnostic de cancer du sein avait été confirmé, et subir un examen de santé mentale et physique. Après avoir obtenu leur consentement, l'auteur s'est entretenu avec chacun des conjoints, pendant que la conjointe recevait un traitement dans un centre médical.

$\mathrm{Au}$ total, vingt participants ont été recrutés dans le cadre de cette étude. Après le $18^{\text {e }}$ entretien, puisqu'aucun nouveau thème ne ressortait des rencontres, il a été établi que la collecte de données avait atteint un point de saturation. L'équipe de recherche a continué de recueillir les données des deux derniers entretiens afin de s'assurer et de confirmer qu'aucun nouveau thème ne ressortirait. La taille des échantillons dans les études phénoménologiques est généralement plus petite, variant de cinq à trente participants (Smith, 2003). 


\section{Collecte de données}

Entre décembre 2018 et août 2019, dans une salle privée de l'hôpital, des entretiens approfondis et non structurés ont été réalisés pour recueillir de l'information au moyen de questions ouvertes comme : «En quoi le cancer de votre conjointe a-t-il affecté votre vie? » et se poursuivaient avec des questions complémentaires (ex. « Dites-m'en plus » et « Donnez-moi un exemple »). Pendant l'entretien, la personne responsable a toujours essayé d'encourager les conjoints à partager leurs expériences de vie avec les patientes. Les rencontres prenaient entre 30 et 60 minutes, selon la tolérance et la coopération des participants, et étaient enregistrées sur support numérique. Les notes d'observation ont été enregistrées par l'intervieweur afin de documenter l'expression et le langage corporel des participants. Conformément aux principes de phénoménologie, les préconceptions de l'équipe de recherche ont été mentionnées, puis laissées de côté dans le but de s'imprégner entièrement de l'expérience des participants (Burkoski et al., 2019; Valle et al., 1989).

\section{Analyse des données}

La collecte et l'analyse des données ont été menées simultanément et, au fur et à mesure que le codage initial était effectué, d'autres entretiens ont été réalisés. Au cours des deux premières étapes, le chercheur tentait d'obtenir une description de l'expérience des conjoints des femmes atteintes d'un cancer du sein. Le processus d'analyse des données s'est inspiré de la méthodologie phénoménologique de Van Manen (1997). La méthode consistait à écouter tous les enregistrements pour se familiarises et s'imprégner de l'expérience des participants. Tous les enregistrements numériques ont été transcrits mot pour mot. Après la transcription, les énoncés thématiques qui ont contribué aux expériences des participants et qui avaient une pertinence directe avec le phénomène ont été extraits en utilisant l'approche interprétative de van Manen (1997). Les déclarations ont mené à des significations, qui ont ensuite été organisées en thèmes communs à tous les participants(Burkoski et al., 2019). Les thèmes ont été développés par le chercheur et revus par l'équipe de recherche.

La structure méthodologique utilisée dans le cadre de cette étude (van Manen, 2016) propose six activités de recherche pour guider la recherche phénoménologique herméneutique (Van Manen, 2014). Le retour continuel à la question de recherche par le chercheur est à la base de ce type d'étude qui utilise la phénoménologie interprétative. La question : « Comment les conjoints des femmes atteintes d'un cancer du sein s'adaptent-ils à leur nouvelle réalité? » a guidé l'extraction et l'interprétation des thèmes à toutes les étapes de l'étude. Finalement, des concepts organisés ont mené à une description détaillée du phénomène.

Le contenu des entretiens transcrits a été analysé en fonction des activités 3 à 6 de l'approche d'analyse thématique de Van Manen. Conformément à l'activité 3, chacun des entretiens transcrits a été considéré dans son ensemble et lu à plusieurs reprises. Une courte description était ensuite écrite afin de résumer l'entretien intégral en quelques phrases ou paragraphes (analyse thématique holistique). Puis, les déclarations thématiques étaient isolées par le biais d’une approche sélective. Pour cette raison, les transcriptions étaient lues sans interruption afin de reconnaître les déclarations qui semblaient particulièrement essentielles et susceptibles de révéler les concepts fondamentaux des expériences adaptatives des hommes face au cancer du sein de leurs conjointes. Ces déclarations étaient alors soulignées. Après avoir extrait les thèmes généraux, les thèmes similaires ont été regroupés.

Puis, en lien avec l'activité 4 , nous avons écrit, puis réécrit pour mettre en mots le phénomène étudié. Pour l'activité 5 , les responsables de l'étude ont cherché à approfondir leur compréhension du phénomène. Dans l'activité 6 , avec la méthode herméneutique, ils se sont référés plusieurs fois à l'ensemble et aux parties du texte pour analyser leurs interconnexions. Finalement, les thèmes extraits ont été modifiés puis reconstruits en fonction de leurs similitudes. Le logiciel MAXQDA a été utilisé pour la gestion des données.

\section{Rigueur de l'étude}

Afin d'assurer une certaine rigueur scientifique, les critères de Lincoln et Guba (1985) à savoir la crédibilité, la transférabilité, la fiabilité et la testabilité ont été appliqués pendant le processus qualitatif. La crédibilité a été assurée par le recrutement de divers individus, en tenant compte de l'âge, de la différence de durée du conflit avec l'expérience (c'est-à-dire le temps écoulé depuis le diagnostic), et les différents services hospitaliers. Pour assurer la transférabilité, des descriptions détaillées des expériences vécues par les conjoints ont été échantillonnées dans deux hôpitaux se trouvant dans des villes différentes. La fiabilité était assurée en suivant de près les procédures d'analyses de données afin d'obtenir des descriptions et transcriptions complètes des expériences des conjoints. Finalement, la testabilité a été obtenue en veillant à ne pas introduire les biais de l'équipe de recherche (pour demeurer ouvert aux partages des participants) et en les excluant tout au long du processus de recherche.

\section{Considérations éthiques}

L'étude a été approuvée par le comité d'éthique de l'Université des sciences médicales de Hamadan (IR.UMSHA. REC.1397.606). Un formulaire de consentement éclairé a été remis à chaque participant. On y expliquait l'objectif de la recherche, les risques potentiels détaillés ainsi qu'une déclaration de confidentialité sur le traitement sécuritaire des renseignements sur les participants.

\section{RÉSULTATS}

\section{Caractéristiques démographiques}

Le groupe était formé de 20 participants âgés de 51 ans, en moyenne. La plupart d'entre eux avaient terminé des études secondaires et supérieures (45\%), habitaient les provinces de Guilan ou de Hamadan et étaient issus de milieux socioéconomiques et ethniques variés. Tous les participants étaient de sexe masculin, de religion islamique et avaient appris le diagnostic de cancer de leur conjointe depuis moins d'un an. Ils étaient tous en santé, vivaient avec leur conjointe, et ils étaient responsables des soins (voir le tableau 1 ). 


\begin{tabular}{|c|c|c|c|c|c|}
\hline Participant no & Âge (années) & Niveau d'éducation & Profession & Lieu de résidence & Origine ethnique \\
\hline 1 & 42 & Diplôme d'études secondaires & Vendeur & Rasht & Farsi \\
\hline 2 & 54 & $9^{e}$ année & Électricien & Rasht & Gilaki \\
\hline 3 & 50 & $5^{e}$ année & Agriculteur & Village & Gilaki \\
\hline 4 & 47 & Diplôme associé & Radiologiste & Anzali & Gilaki \\
\hline 5 & 50 & École primaire & Vitrier & Rasht & Gilaki \\
\hline 6 & 55 & Analphabète & Manoeuvre & Village & Gilaki \\
\hline 7 & 49 & École primaire & Chauffeur de taxi & Rasht & Turc \\
\hline 8 & 50 & Diplôme d'études secondaires & $\begin{array}{l}\text { Peintre en bâtiment, } \\
\text { théâtre, entraîneur }\end{array}$ & Rasht & Farsi \\
\hline 9 & 54 & Analphabète & Manœuvre (Plombier) & Village & Gilaki \\
\hline 10 & 55 & Diplôme associé & $\begin{array}{l}\text { Employé Fondation des } \\
\text { anciens combattants }\end{array}$ & Hamadan & Farsi \\
\hline 11 & 45 & Baccalauréat & $\begin{array}{c}\text { Travailleur dans une société } \\
\text { pharmaceutique }\end{array}$ & Rasht & Gilaki \\
\hline 12 & 57 & École primaire & $\begin{array}{l}\text { Travailleur de la } \\
\text { construction }\end{array}$ & Village & Gilaki \\
\hline 13 & 67 & École primaire & Agriculteur, arboriculteur & Village & Mazani \\
\hline 14 & 70 & Baccalauréat & Retraité de l'armée de l'air & Banlieue de Hamadan & Turque \\
\hline 15 & 32 & Baccalauréat & Vendeur de rideaux & Rasht & Gilaki \\
\hline 16 & 44 & Baccalauréat & Salarié & Hamadan & Farsi \\
\hline 17 & 62 & Diplôme d'études secondaires & Retraité & Hamadan & Farsi \\
\hline 18 & 54 & Analphabète & Agriculteur & Banlieue de Hamadan & Turc \\
\hline 19 & 57 & 10e année & Commerçant & Hamadan & Farsi \\
\hline 20 & 49 & École primaire & Manoeuvre & Village & Gilaki \\
\hline
\end{tabular}

\section{Thèmes ressortis}

L'analyse des expériences des participants a fait ressortir cinq thèmes : la souffrance émotionnelle, le fardeau des soins, le maintien de la cohésion familiale, le caractère incertain de l'avenir et le sentiment de perte du concept de soi. Le concept d'adaptation a été défini en fonction de ces thèmes et des expériences des conjoints. Il considère l'évolution inconnue de la maladie et le besoin de répit découlant du sentiment de perte de concept de soi et des efforts déployés pour préserver la cohésion familiale en endurant la souffrance émotionnelle et le fardeau des soins. Le tableau 2 présente les thèmes extraits des données qualitatives. Voici des expériences de participants découlant de ces thèmes :

Thème 1: Souffrance émotionnelle

Ce thème englobe trois sous-thèmes : le sentiment d'être délaissé et le besoin de répit, l'instabilité émotionnelle du conjoint, et l'obligation de composer avec les sentiments et les émotions des patientes. Puisque les femmes ont constamment besoin de l'attention et des soins de leur conjoint, la plupart d'entre eux se sentent négligés, disent ne plus s'amuser, ne plus voir leurs amis, ni faire leurs activités, ce qui accroît leur sentiment de solitude. Les conjoints sont confrontés à
Tableau 2 : Thèmes extraits à partir des données qualitatives

\begin{tabular}{|l|l|}
\hline Thème principal & Sous-thème \\
\hline $\begin{array}{l}\text { Souffrance } \\
\text { émotionnelle }\end{array}$ & $\begin{array}{l}\text { Délaissement, répit, instabilité émotionnelle } \\
\text { du conjoint } \\
\text { Faire face aux émotions des patientes }\end{array}$ \\
\hline $\begin{array}{l}\text { Le fardeau des soins } \\
\text { et du traitement }\end{array}$ & $\begin{array}{l}\text { La pression des services médicaux } \\
\text { inadéquats, conjoint négligé par l'équipe } \\
\text { médicale, endurer les tensions liées au } \\
\text { travail et au coût de la vie } \\
\text { Assumer plusieurs rôles }\end{array}$ \\
\hline $\begin{array}{l}\text { Maintien de la } \\
\text { cohésion familiale }\end{array}$ & $\begin{array}{l}\text { Transition du déni à l'acceptation, progrès } \\
\text { du conjoint, stratégies d'adaptation du } \\
\text { conjoint, impact sur les interactions } \\
\text { personnelles, familiales et sociales } \\
\text { Froideur de la relation }\end{array}$ \\
\hline Avenir incertain & $\begin{array}{l}\text { Caractère incertain du cancer, alternance } \\
\text { entre l'espoir et le désespoir }\end{array}$ \\
\hline $\begin{array}{l}\text { Sentiment de perte } \\
\text { du concept de soi }\end{array}$ & $\begin{array}{l}\text { Tourments physiques du conjoint, impact } \\
\text { sur le moral du conjoint }\end{array}$ \\
\hline
\end{tabular}


leurs propres vagues à l'âme sentimentaux et émotionnels et aux fluctuations équivalentes de leur conjointe, oscillant entre l'espoir et le désespoir.

\section{Participant $\mathrm{n}^{\circ} 10$}

"J'ai cessé toutes les tâches que je faisais d'habitude, même de m'occuper de mon beau jardin, parce que je n'y arrivais plus. J'ai une plantation d'orangers qui a été infestée d'insectes parce que je n'ai pas pu les vaporiser. Je ne m'amuse plus, je ne discute plus qu'avec elle. »

\section{Participant $\mathrm{n}^{\circ} 4$}

"On se demande toujours si on en a fait assez et si quelque chose arrivera. Il faut faire de notre mieux, mais c'est stressant. Qu'est-ce qu'on a réussi à faire et qu'est-ce que ça donne? Parce qu'on se demande toujours ce qui va se passer. On se sent coupable si on pense ne pas avoir fait ce qu'il fallait. »

\section{Participant $\mathrm{n}^{\circ} 1$}

«C'était difficile. Ma femme avait des problèmes psychologiques, et puisque personne ne pouvait m'aider pour son traitement, j'ai dû l'envoyer rester chez son père. Elle avait de la douleur et avait besoin de repos. Elle va mieux maintenant, mais à cause de ses médicaments, c'était très difficile au début, quand elle avait des problèmes de santé mentale. »

Thème 2: Assumer le fardeau des soins et des traitements

Ce thème comprend quatre sous-thèmes : la pression causée par des services médicaux inadéquats, la négligence de l'équipe médicale à l'égard de la patiente, les pressions liées au travail et aux moyens de subsistance ainsi que les nombreux rôles à assumer.

En raison de la maladie, les conjoints des patientes sont confrontés à certains problèmes comme le manque de soutien des compagnies d'assurance, le temps requis pour se procurer les médicaments, le manque de confiance envers ces médicaments et leur coût exorbitant, l'accès insuffisant à des soins médicaux et des soins appropriés, l'accès limité à l'information et l'absence d'une équipe médicale efficace. Pour résoudre ces problématiques, il faudrait un effort soutenu de la part de tous les professionnels médicaux. Dans certains cas, les conjoints se sont plaints d'être ignorés par l'équipe soignante, dénonçant les décisions « patriarcales » de l'équipe médicale et le manque d'attention accordée aux besoins de base de ce groupe. Le rôle traditionnel des femmes iraniennes (qui sont mères au foyer, s'occupent des tâches domestiques et de la famille et se concentrent sur les enfants et leur bonheur) devient plus passif une fois qu'elles tombent malades. Par conséquent, ces responsabilités incombent aux hommes et s'ajoutent aux rôles de conjoint et de père. Les conjoints se retrouvent seuls à tout gérer.

\section{Participant $\mathrm{n}^{\circ} 4$}

«Un patient avec une maladie bien précise n'a pas nécessairement accès à tout. Avec l'équipe médicale par exemple: après la chimiothérapie, on ne savait pas quels aliments elle devait manger. Mais il n'y avait pas de nutritionniste dans léquipe. On ne savait pas quoi faire quand un symptôme apparaissait, parce que personne n'était là pour nous renseigner. Il n'y avait pas de psychologue, ni de nutritionniste, ni d'oncologue dans léquipe. Tout cela nous a beaucoup insécurisés. »

Participant $\mathrm{n}^{\circ} 7$

«On ne demande pas l'avis du conjoint pour le traitement. L'hôpital ne m'a pas demandé mon consentement quand ils ont voulu opérer.

Intervieweur : "Vous voulez dire que vous ne le saviez pas?

Conjoint: Je le savais, mais ils ont fait la mastectomie sans mon consentement. »

Participant $\mathrm{n}^{\circ} 11$

"C'est épouvantable. Il n'y a aucun soutien financier. Certaines familles n'ont rien, et les pauvres ont du mal à trouver des petites sommes. Il $y$ a un problème avec la radiothérapie; les gens veulent passer au public, mais l'offre ne suffit pas à la demande. D'un autre côté, les frais au privé sont très élevés et ne sont pas couverts par les assurances. Certaines personnes ne sont couvertes que par les services sociaux et médicaux et doivent débourser de 10 à 15 millions de tomans. »

Participant $\mathrm{n}^{\circ} 6$

«Ce n'était pas comme ça avant. En rentrant du travail, j'allais me coucher. Mais je ne peux plus faire ça maintenant puisque je dois faire la plupart des tâches : laver ses vêtements, préparer ses repas et faire son lit, l'amener chez le médecin et acheter ses médicaments. Je ne le faisais pas avant, mais maintenant je dois le faire. »

\section{Thème 3 : Préserver la cohésion familiale}

Ce thème comprend cinq sous-thèmes : la transition du déni à l'acceptation, les progrès du conjoint, les stratégies d'adaptation du conjoint, la détérioration des interactions personnelles, familiales et sociales et la froideur de la relation. Tous les conjoints ont connu les périodes de transition du déni vers l'acceptation de la nouvelle situation, et chacun a vécu différemment ces périodes. Éventuellement, ils ont utilisé des stratégies d'adaptation pour se remonter le moral, et celui des patientes. Avec toutes ces stratégies nécessaires pour s'adapter aux problèmes, tous les participants considèrent que leurs relations interpersonnelles, familiales et sociales ont été affectées par la maladie et ses conséquences.

Participant $\mathrm{n}^{\circ} 5$

"Pour faire plaisir à ma femme, nous sommes allés à quelques mariages, soirées et fêtes d'anniversaire. Hier soir, nous avons invité 10 personnes à la maison pour la fête de ma fille. On essaie de garder le moral. »

Participant $\mathrm{n}^{\circ} 14$

«Un psychanalyste est aussi présent lors des séances. On discute, et ça m'aide à me calmer. Je fais aussi de l'écriture et de la peinture. »

Participant $\mathrm{n}^{\circ} 4$ «On était très tendus après la chimiothérapie. Nous l'étions tous, étant donné que les membres de la famille ne doivent 
pas attraper de rhume ou tomber malades pour protéger la patiente. Plusieurs membres de la famille ne peuvent pas entrer en contact avec nous, alors on reste seul, avec la maladie et la patiente. »

Participant $\mathrm{n}^{\circ} 10$

« Je ne peux plus avoir ce genre de relation émotionnelle et affective, et je m'y fais. De temps en temps, j'essaie d'entretenir la flamme. J'essaie de ne pas laisser le fossé se creuser davantage, de ne pas en être la cause. La maladie a déjà fait assez de ravages. »

\section{Thème 4 : Avenir incertain}

Ce thème regroupe deux sous-thèmes : caractère incertain du cancer et alternance entre l'espoir et le désespoir. La plupart des conjoints ont parlé de la peur constante du mot « cancer » qu'ils avaient avant que leur conjointe développe la maladie. Après le diagnostic, ils avaient toujours peur de perdre leur conjointe, et envisageaient l'avenir tantôt avec espoir, tantôt avec appréhension. Ils ignorent ce qu'il adviendra avec la maladie et le traitement.

\section{Participant $\mathrm{n}^{\circ} 4$}

«Avoir confiance en Dieu suffit. Ce sont là de belles paroles, mais ça ne reflète pas la vraie vie. Ce n'est pas si simple de s'en remettre à Dieu quand cela concerne la personne avec qui on vit depuis des années. On s'inquiète toujours de ce qui pourrait arriver. »

\section{Thème 5 : Le sentiment de perte du concept de soi}

Ce thème comprend deux sous-thèmes : les tourments physiques du conjoint et leurs répercussions sur son moral. La plupart des conjoints de patientes ayant un cancer du sein ont dit être constamment préoccupés par l'échec du traitement et se sont plaints du fardeau ainsi que de la pression reliés aux soins, qui leur causent plusieurs atteintes physiques et psychologiques. Létat de la patiente force le conjoint à s'oublier et à ignorer ses propres besoins.

\section{Participant $\mathrm{n}^{\circ} 12$}

"J'ai moi-même été malade et ressenti des sensations inhabituelles sur mon visage pendant un moment. Je me réveillais la nuit avec un mal de tête, alors je suis allé voir un médecin et j'ai eu une scintigraphie du cerveau. Heureusement, il a dit qu'il n'y avait rien, seulement une tension nerveuse, et il m'a prescrit des médicaments. Je n'ai rien dit à personne parce que je n'avais pas le choix, je ne parlais à personne. »

\section{DISCUSSION}

Bien que plusieurs études aient été réalisées pour comprendre les divers impacts du cancer du sein sur les conjoints des patientes, peu d'études ont tenté de clarifier les expériences de ces hommes dans la société iranienne. Les résultats de la présente étude phénoménologique indiquent que le concept d'adaptation vécue en tant que conjoint d'une femme atteinte d'un cancer du sein considère le caractère incertain de l'avenir, le sentiment de perte de l'image de soi ainsi que la responsabilité des conjoints de préserver la cohésion familiale, de soutenir la souffrance émotionnelle et d'assumer la charge des soins aux patientes. En tant qu'aidants principaux des patientes dans le processus de traitement et de rétablissement, les conjoints peuvent jouer un rôle important dans l'accompagnement des patientes et de l'équipe médicale. Comprendre le concept d'adaptation du point de vue des conjoints de femmes luttant contre le cancer du sein et les aider à mieux s'adapter à la situation influence grandement le traitement et le rétablissement des patientes.

La souffrance émotionnelle constitue un résultat important de l'étude. Ces conjoints ont dit souffrir de solitude et se sentir malheureux sans un soutien adéquat de la part du personnel médical et de leur famille. De plus, les fluctuations comportementales et émotionnelles des patientes atteignent les conjoints, qui vivent eux aussi cette instabilité. Plusieurs études font état d'un déséquilibre et d'une détresse émotionnelle, tant chez les patientes que chez leurs conjoints (Charvoz et al., 2016; Duprez et al., 2017; Zimmermann, 2015). Dans une étude qualitative menée en Turquie, Çömez et Karayurt (2016) ont comparé le vécu des conjoints face au cancer du sein de leur femme à une crise, alors qu'ils décrivaient leurs inquiétudes et leur peur de la mort. Dans une autre étude, Hasson-Ohayon et collaborateurs (2010) ont mis l'accent sur le désespoir émotionnel, la dépression et l'anxiété des conjoints et signalé un faible soutien des conjoints par leurs familles et amis au cours de ce processus.

Le fardeau des soins et des traitements est un autre important constat de la présente étude portant sur les conjoints de femmes atteintes d'un cancer du sein, qui font face à de nombreux problèmes financiers en raison du coût des traitements et de l'incapacité des patientes à générer des revenus. Dans bien des cas, cette perte de salaire augmente considérablement la pression ressentie par les hommes. Plusieurs études reconnaissent la pression financière des conjoints de patientes atteintes du cancer du sein (Glajchen, 2004; Grunfeld et al., 2004; Weitzner et al., 1999). Parmi les autres difficultés des conjoints et soignants iraniens, citons le coût élevé des traitements, l'absence d'installations de radiothérapie dans tous les centres, la défaillance ou le manque d'installations nécessaires pour le grand nombre de patients référés, la forte concentration des meilleurs médecins dans la capitale (qui entraîne un manque d'accès aux spécialistes dans toutes les sphères de la société), la pénurie et l'inquiétude liées aux médicaments, le manque de soutien des compagnies d'assurance et l'absence d'une seule et même équipe médicale pour le traitement. Selon la définition sur l'équité dans les soins de santé, soit la distribution équitable des services médicaux entre les personnes et les différentes classes sociales (Zere et al., 2007), il s'agit de l'un des principaux enjeux auxquels se heurtent les conjoints de femmes atteintes d'un cancer du sein en Iran.

Les conjoints de femmes ayant un cancer du sein font tout leur possible pour préserver la cohésion familiale. Les nombreuses difficultés qu'ils rencontrent, comme les problèmes financiers ou médicaux, les changements physiques et comportementaux de leurs épouses et la pression qui retombe sur la famille perturbent la cohésion familiale et les relations. En tant que chef de famille, le conjoint doit redoubler ses efforts 
pour remédier à la situation. Dans la présente étude, tous les participants ont reconnu l'importance de préserver la cohésion familiale. Pour les conjoints, leur rôle le plus important était de faire en sorte que leur conjointe garde le moral, et tous s'entendaient pour dire que cela aide à mieux affronter la maladie. Dans leurs études, Neris et Anjos (2014) et Zahlis et Lewis (2010) ont mentionné les efforts des conjoints pour conserver l'unité familiale, ce qui correspond aux résultats de la présente étude.

L'expérience des Iraniens qui ont participé à cette étude témoigne du caractère incertain de l'avenir et de l'appréhension constante des femmes par rapport aux résultats de leur traitement et des conséquences de leur maladie. Malgré tous les efforts des conjoints pour la survie des patientes, le fait de ne pas savoir ce qui les attend jette une ombre sur leur vie. De nombreuses études ont reconnu cette problématique (Gao et al., 2019; Ghaemi et al., 2019; Younes Barani et al., 2019).

Les conjoints de femmes ayant un cancer du sein sont aussi confrontés au sentiment de perte du concept de soi, étant forcés de mettre de côté les différents aspects de leur vie personnelle pour s'acquitter de diverses tâches et assumer leurs nouveaux rôles imposés par la maladie. Plusieurs activités, relations amicales et loisirs sont considérablement restreints ou inexistants pour ces hommes qui éprouvent divers problèmes physiques et mentaux en raison de ces tensions additionnelles. Par conséquent, Girgis et collaborateurs (2013) se sont intéressés à l'incidence du cancer sur les proches aidants, plus précisément sur les troubles physiques, mentaux et sociaux engendrés par la maladie, et son impact sur les relations et le stress financier. Compte tenu de la culture iranienne axée sur la religion et du rôle de soutien attendu des hommes dans la famille, il convient de se pencher sur la manière dont les conjoints s'adaptent à la maladie de leur femme. Cette démarche pourrait représenter une stratégie de résolution de problème pour les personnes atteintes de cancer et ainsi améliorer les soins aux patients, faciliter l'adaptation à la maladie et favoriser une ambiance familiale sereine.

\section{Limites}

Les limites de la présente étude reposent sur la généralisation limitée des données, ce qui survient dans les études interprétatives phénoménologiques de type herméneutique en raison du caractère « personnel » des données obtenues à

\section{RÉFÉRENCES}

Abachizadeh, K., Moradi-Kouchi, A., Ghanbari-Motlagh, A., Kousha, A., Shekarriz-Foumani, R., \& Erfani, A. (2018). Breast cancer in Iran: Levels, variations and correlates. Community Health (Salāmat-i ijtimā̄̄), 5(1), 11-21.

Braun, M., Mikulincer, M., Rydall, A., Walsh, A., \& Rodin, G. (2007). Hidden morbidity in cancer: spouse caregivers. Journal of Clinical Oncology, 25(30), 4829-4834.

Bray, F., Ferlay, J., Soerjomataram, I., Siegel, R. L., Torre, L. A., \& Jemal, A. (2018). Global cancer statistics 2018: GLOBOCAN estimates of incidence and mortality worldwide for 36 cancers in 185 countries. CA: a cancer journal for clinicians, 68(6), 394-424.

Burkoski, V., Yoon, J., Hutchinson, D., Solomon, S., \& Collins, B. E. (2019). Experiences of Nurses Working in a Fully Digital partir d'expériences vécues. Afin de surmonter cette limite, les expériences de participants de deux régions en Iran ont été utilisées. Toutefois, les résultats de la présente étude sont seulement applicables à l'Iran, puisque l'adaptation des conjoints de femmes ayant un cancer du sein peut être différent dans les autres pays. En outre, en raison du contexte traditionnel de la population et de la probabilité que les participants aient été contraints par certaines normes islamiques et le sexe de la personne passant l'entrevue, les participants pourraient ne pas avoir été à l'aise de discuter de certains enjeux. Le chercheur a fait de son mieux pour rassurer les participants et étudier en profondeur le phénomène d'adaptation.

L'un des points forts de cette étude réside dans la méthode et l'approche utilisées pour ne pas dénaturer l'expérience des participants. Les efforts mis sur l'attention et l'aide apportées aux conjoints des femmes atteintes d'un cancer du sein pour les aider à faire face à la maladie peuvent servir les établissements et le personnel soignant. Il est également recommandé que d'autres études soient réalisées afin de produire des instruments de mesure de l'adaptation chez les conjoints de femmes atteintes d'un cancer du sein qui seront utiles en Iran.

\section{CONCLUSION}

Les résultats de cette étude portant sur l'expérience des conjoints de femmes touchées par un cancer du sein font ressortir leurs difficultés d'adaptation à la situation. Ce processus d'adaptation se décline en cinq thèmes : la souffrance émotionnelle, le fardeau des soins, le maintien de la cohésion familiale, le caractère incertain de l'avenir et le sentiment de perte du concept de soi. Le problème d'adaptation des Iraniens dont la femme souffre d'un cancer du sein revêt un caractère multidimensionnel qui peut se manifester différemment dans d'autres pays en raison des différences dans les aspects culturels et les systèmes de soins de santé. Par conséquent, nous pensons que ces hommes peuvent mieux affronter la situation en comprenant la notion d'adaptation et en abordant cette question par le biais de stratégies de santé. Ainsi, des mesures peuvent être prises afin d'assurer des soins médicaux adéquats aux patientes et à leurs conjoints. Il est également conseillé d'accorder une attention particulière aux conjoints de patientes atteintes de cancer et à leur capacité d'adaptation face à la situation afin de créer des conditions qui favorisent l'adaptation à cet enjeu.

Hospital: A Phenomenological Study. Canadian Journal of Nursing Leadership, 32(Sp), 72-85.

Cebeci, F., Yangın, H. B., \& Tekeli, A. (2012). Life experiences of women with breast cancer in south western Turkey: A qualitative study. European Journal of Oncology Nursing, 16(4), 406-412.

Charvoz, L., Favez, N., Notari, S. C., Panes-Ruedin, B., \& Delaloye, J.-F. (2016). A survey of couples facing breast cancer in women. In C. R. In M. Oris, D. Joye, \& M. E. Stähli (Ed.), Surveying human vulnerabilities across the life course (Vol. 3, pp. 113-129): Springer, Cham.

Çömez, S., \& Karayurt, Ö. (20I6). We as spouses have experienced a real disaster!: A qualitative study of women with breast cancer and their spouses. Cancer nursing, 39(5), E19-E28. 
Dorval, M., Guay, S., Mondor, M., Masse, B., Falardeau, M., Robidoux, A., . . Maunsell, E. (2005). Couples who get closer after breast cancer: frequency and predictors in a prospective investigation. Journal of Clinical Oncology, 23(15), 3588-3596.

Duprez, C., Vanlemmens, L., Untas, A., Antoine, P., Lesur, A., Loustalot, C., ... Carlier, D. (2017). Emotional distress and subjective impact of the disease in young women with breast cancer and their spouses. Future Oncology, 13(29), 2667-2680.

Ferlay, J., Steliarova-Foucher, E., Lortet-Tieulent, J., Rosso, S., Coebergh, J.-W. W., Comber, H., ... Bray, F. (2013). Cancer incidence and mortality patterns in Europe: estimates for 40 countries in 2012. European journal of cancer, 49(6), 1374-1403.

Fink, G. (2016). Stress: Concepts, cognition, emotion, and behavior: Handbook of stress series (Vol. 1): Academic Press.

Folkman, S., \& Lazarus, R. S. (1988). Manual for the ways of coping questionnaire. Palo Alto: CA: Consulting Psychologists Press.

Gao, L., Liu, J. E., Zhou, X. P., Su, Y. L., \& Wang, P. L. (2019). Supporting her as the situation changes: A qualitative study of spousal support strategies for patients with breast cancer in China. European journal of cancer care, 29(1), e13176.

Ghaemi, S. Z., Keshavarz, Z., Tahmasebi, S., Akrami, M., \& Heydari, S. T. (2019). Conflicts women with breast cancer face with: A qualitative study. Journal of family medicine and primary care, $8(1)$, 27.

Ghaffari, F., Shali, M., Shoghi, M., \& Joolaee, S. (2014). Psychometric properties of the persian version of the self-assessed support needs questionnaire for breast cancer cases. APJCP, 15(3), 1435-1440.

Girgis, A., Lambert, S., Johnson, C., Waller, A., \& Currow, D. (2013). Physical, psychosocial, relationship, and economic burden of caring for people with cancer: a review. Journal of Oncology Practice, 9(4), 197-202.

Glajchen, M. (2004). The emerging role and needs of family caregivers in cancer care. The journal of supportive oncology, 2(2), 145-155.

Grunfeld, E., Coyle, D., Whelan, T., Clinch, J., Reyno, L., Earle, C. C.,... Janz, T. (2004). Family caregiver burden: results of a longitudinal study of breast cancer patients and their principal caregivers. Cmaj, 170(12), 1795-1801.

Hasson-Ohayon, I., Goldzweig, G., Braun, M., \& Galinsky, D. (2010). Women with advanced breast cancer and their spouses: diversity of support and psychological distress. Psycho-oncology, 19(11), 1195-1204.

Kershaw, T., Northouse, L., Kritpracha, C., Schafenacker, A., \& Mood, D. (2004). Coping strategies and quality of life in women with advanced breast cancer and their family caregivers. Psychology Q Health, 19(2), 139-155. doi: 10.1080/08870440310001652687

Lopes, V. B., Lobo, A. P. A., Da Silva Junior, G. B., Melo, A. K., Lamboglia, C. G., \& Silva, C. A. B. d. (2018). The experience of male spouses in the context of breast cancer: a systematic review of the literature. Psychology, health a medicine, 23(1), 89-98.

Mehrabi, E., Hajian, S., Simbar, M., \& Hoshyari, M. (2016). Religion, spirituality and coping with breast cancer: A phenomenological study. Journal of Research on Religion Q Health, 2(2), 12-23.

Momenimovahed, Z., \& Salehiniya, H. (2019). Epidemiological characteristics of and risk factors for breast cancer in the world. Breast Cancer: Targets and Therapy, 11, 151-164.

Montazeri, A., Vahdaninia, M., Harirchi, I., Harirchi, A. M., Sajadian, A., Khaleghi, F., ... Jarvandi, S. (2008). Breast cancer in Iran: need for greater women awareness of warning signs and effective screening methods. Asia Pacific family medicine, 7(1), 6.

Nafissi, N., Khayamzadeh, M., Zeinali, Z., Pazooki, D., Hosseini, M., \& Akbari, M. E. (2018). Epidemiology and histopathology of breast cancer in Iran versus other Middle Eastern countries. Middle East Journal of Cancer, 9(3), 243-251.
Nasiri, A., Taleghani, F., \& Irajpour, A. (2016). Adjustment process in I ranian men to their wives' breast cancer. European journal of cancer care, 25(2), 307-317.

Neris, R. R., \& Anjos, A. C. Y. d. (2014). Experience of spouses of women with breast cancer: an integrative literature review. Revista da Escola de Enfermagem da USP, 48(5), 922-931.

Northouse, L. L., Mood, D., Templin, T., Mellon, S., \& George, T. (2000). Couples' patterns of adjustment to colon cancer. Social science Q medicine, 50(2), 271-284.

Paley, J. (2018). Meaning, lived experience, empathy and boredom: Max van Manen on phenomenology and Heidegger. Nursing Philosophy, 19(3), e12211.

Sajadian, A., Heydari, L., \& Mokhtari, P. (2015). Investigating Care Problems in Family Care Care Patients with Breast Cancer. Iranian Journal of Breast Disease Research, 8(2), 7-14.

Smith, J. A. (2003). Qualitative psychology: A practical guide to research methods: Sage Publications, Inc.

Soothill, K., Morris, S., Thomas, C., Harman, J., Francis, B., \& McIllmurray, M. (2003). The universal, situational, and personal needs of cancer patients and their main carers. European Journal of Oncology Nursing, 7(1), 5-13.

Valle, R. S., King, M., \& Halling, S. (1989). An introduction to existential-phenomenological thought in psychology. In I. R. S. Valle \& S. Halling (Eds.), Existential-phenomenological perspectives in psychology (pp. 3-16): Springer.

van Manen, M. (2016). Researching lived experience: Human science for an action sensitive pedagogy: Routledge.

Walker, W. (2007). Ethical considerations in phenomenological research. Nurse researcher, 14(3), 36-45.

Weitzner, M. A., Jacobsen, P., Wagner, H., Friedland, J., \& Cox, C. (1999). The Caregiver Quality of Life Index-Cancer (CQOLC) scale: development and validation of an instrument to measure quality of life of the family caregiver of patients with cancer. Quality of life Research, 8(1-2), 55-63.

Xiong, M., Stone, T. E., Turale, S., \& Petrini, M. A. (2016). Women's experiences of making healthcare decisions about their breast cancer: A phenomenological study. Nursing \& health sciences, 18(3), 314-320.

Yeung, N. C., Zhang, Y., Ji, L., Lu, G., \& Lu, Q. (2018). Guilt among husband caregivers of Chinese women with breast cancer: The roles of male gender-role norm, caregiving burden and coping processes. European journal of cancer care, 27(5), e12872.

Younes Barani, Z., Rahnama, M., Naderifar, M., Badakhsh, M., \& Noorisanchooli, H. (2019). Experiences of Spouses of Women with Breast Cancer: A Content Analysis. Asian Pacific Journal of Cancer Prevention, 20(10), 3167-3172.

Yusoff, N., Reiko Yap, K., \& Ahmad, A. (2012). Husbands' experience with their wives' breast cancer: a qualitative study. Malaysian Journal of Public Health Medicine, 12(1), 31-38.

Zahlis, E. H., \& Lewis, F. M. (2010). Coming to grips with breast cancer: the spouse's experience with his wife's first six months. Journal of psychosocial oncology, 28(1), 79-97.

Zere, E., Mandlhate, C., Mbeeli, T., Shangula, K., Mutirua, K., \& Kapenambili, W. (2007). Equity in health care in Namibia: developing a needs-based resource allocation formula using principal components analysis. International Journal for Equity in Health, 6(1), 3.

Zimmermann, T. (2015). Intimate relationships affected by breast cancer: interventions for couples. Breast Care, 10(2), 102-108. 